\title{
Enterprise Microblogging
}

\section{DOI 10.1007/s12599-010-0129-1}

\section{The Authors}

PD Dr. Kai Riemer ( $\varangle)$

Discipline of Business Information

Systems

Faculty of Economics \& Business

University of Sydney

Building $\mathrm{H} 69$

Sydney

NSW 2006

Australia

kai.riemer@sydney.edu.au

\section{Dr. Alexander Richter}

Institut für Softwaretechnologie

Fakultät für Informatik

Universität der Bundeswehr München

85577 Neubiberg

Germany

a.richter@unibw.de

Dipl.-Wirt.-Inf. Martin Bohringer

Professur für Wirtschaftsinformatik II, insb. Systementwicklung/

Anwendungssysteme

Fakultät für Wirtschaftswissenschaften

Technische Universität Chemnitz

09126 Chemnitz

Germany

martin.boehringer@wirtschaft.

tu-chemnitz.de

Received: 2010-04-12

Accepted: 2010-08-11

Accepted after two revisions by

Prof. Dr. Sinz.

Published online: 2010-10-16

This article is also available in German in print and via http://www. wirtschaftsinformatik.de: Riemer K, Richter A, Böhringer M (2010) Enterprise Microblogging. WIRTSCHAFTSINFORMATIK. doi: 10.1007/s11576010-0246-y.

(c) Gabler Verlag 2010

\section{Microblogging as a Form of Corporate Communication}

ICT support of communication in organizations is not only becoming more important, but also more difficult. For example, many knowledge workers increasingly experience information overload with regard to their email inboxes, as many users have fallen into the habit of sending messages to large numbers of receivers simultaneously (using the CC feature). Typical reasons for such behavior are that users want to reassure others, that they do not know for whom a particular piece of information might be relevant, or just habit. As a result, a majority of email users today is confronted with an unsorted mass of messages, of which only a fraction is actually relevant for them personally. The sorting and processing of these messages on the other hand is time consuming and cumbersome; moreover, the likelihood increases that the user overlooks important information in the flood of messages. Against this backdrop, the principle of Enterprise Microblogging (EMB) promises to create a new channel for organizational and team communication. Similar to Twitter on the public Internet, users can post short messages in this new channel, which is especially beneficial in cases where information may be relevant for a whole group of users that is unknown in advance. For example, users might post links to interesting online resources, report on important events or just update others on team tasks they have finished. The resulting information stream can then be composed and filtered by the platform users in various ways, in order to ensure relevance of what messages are personally received. Besides, all messages are stored and remain accessible and searchable for future reference.

Initial case studies have shown that successful EMB platforms have the potential to shift communication formerly conducted via email to a new public communication space facilitated by microblogging (Barnes et al. 2010; Riemer and Richter 2010). While such a step yields a range of opportunities, e.g., for corporate innovation and knowledge management, it is not without challenges with regard to changes in individual and group communication behavior, for both management and users.

\section{What is Microblogging?}

\subsection{The Emerging Twitter Phenomenon}

Microblogging is a communication service which enables users to post short messages into an initially undirected message stream. Users can then subscribe to other users' messages, so that a directed information stream is created. The most prominent microblogging service is Twitter, launched in 2006, which allows its users to post messages restricted to 140 characters. While many similar services have emerged in the market, Twitter with its philosophy and features still shapes the public perception of microblogging, which becomes evident in the meanwhile common terms "tweeting" and "tweet".

Beside the restriction of message length, Twitter has introduced the "following" principle as another distinctive feature which sets it apart from other social software applications. On Twitter, the messages posted by its users in the first instance create an undirected real-time information stream. Only by "following" other users and thereby subscribing to their messages does a user create a personalized information stream, which is then presented on the user's start page in chronological order. Whereas platforms such as Facebook and Xing require both users to confirm a connection, Twitter's social graph, which is created by the "following" mechanism, also shows unidirectional connections (user A follows user B, but user B does not follow user A).

Features, such as referencing other users using the @user tag, the referencing of other users' posts (retweeting), or the tagging of message with the hashtag (\#keyword), which are a core part of the platform today, developed from user appropriations (users started including these tags in their messages) and were only later included in the user interface of the platform (Java et al. 2007).

Apart from this user-driven evolution, Twitter offers open access to its core functionalities via an API which also introduces innovation to the service. Consequently, the Twitter API is the basis for thousands of third party applications which complement the service with special features. Most notably, applications 
for mobile devices, by using the API, account for the majority of tweets posted to the platform today. Henceforth, Twitter has achieved almost ubiquitous diffusion; it is now possible to use the service independent of space and time, and from almost any device.

\subsection{Typical Usage Patterns}

When launching Twitter, the company tried to frame the usage of its platform with the motto "What are you doing?". However, in the meantime it has turned out that only a minority of user posting fall into this category, a fact that is well in line with the above mentioned user appropriation (Java et al. 2007). As a consequence, the motto today reads in more general terms: "What's happening?". Not surprisingly, a first set of studies have taken a closer look at user communication behavior on the platform. According to a recent study, the most common types of communication are Me Now (41\%: "tired and upset"), Statements and Random Thoughts (25\%: "The sky is blue in the winter here"), and Opinions/Complaints (24\%: "Go Aussie \$ go!"), while true Information Sharing ("15 Uses of WordPress <URL REMOVED>") only happens in $21 \%$ of all posts (Naaman et al. 2010). On the other hand, the results also show that the service has been appropriated for very different purposes and by different target groups, for both hedonic and professional communication.

\section{Enterprise Microblogging}

\subsection{Potentials and Reservations}

Due to its simplicity and immediacy, microblogging is also of interest to corporations, e.g., to support communication and coordination in teams and projects. Consequently, the microblogging principle has been adapted to the enterprise context. Today, four years after the launch of Twitter, a range of EMB platforms have been launched, but platform offerings appear to still outstrip demand for the service.

When marketing Enterprise Microblogging to businesses, one of the key features of the service seems to act as a main barrier: Twitter as an open platform is largely shaped by the interest and behavior of its users. That is why many decision makers hold strong reservations against the application of microblogging; many fear that in transferring this technology from the public Internet into the Intranet they will also import the above-described communication behavior, which is associated with unproductive "chatter" and procrastination. On the other hand, many decision makers also contemplate if they should forego the often-discussed potentials of microblogging for group communication, or open their organizations to this new medium.

\subsection{Platforms and Technical Proliferation}

Due to the above-mentioned openness ("Nutzungsoffenheit") of such platforms, many EMB providers have focused their applications and subsequently tailored their products to fit certain usage scenarios. For example, tools exist for project and information management which put emphasis on open communication and elaborate filtering and analysis features, but at the same time the follower principle is omitted, as communication takes place in stable groups (i.e., project teams). Other providers focus on large organizations that want to facilitate social networking among their employees; hence, the follower mechanism becomes more important, while special features, e.g., for information management, become less dominant in favor of simplicity and ease-of-use.

Similar to the variety in features, a range of different delivery modes exists, which interested user organizations have to take into account in making a decision: open source, software-as-a-service (SaaS), and traditional license models exist. Typical EMB service providers are StatysNet (open source), Communote, Present.ly, Socialcase, Socialtext, and Yammer (commercial). A market overview can be found in Böhringer et al. (2010). On the technical level, according to market analysts, a widespread integration of microblogging features into existing IT infrastructures is imminent (Gartner 2010).

\section{A Use Case}

In the following we will illustrate the potential of Enterprise Microblogging for team and project work using a case example (cf. Riemer and Richter 2010).
The case company is a medium-sized software provider that has developed its own EMB platform which it offers to the market, but also uses internally. The above-quoted study investigated usage practices in a software development team. When doing so, it used genre analysis to identify usage patterns, which allow reflecting on the role of the platform in the context of project work.

One main motivation for introducing the EMB platform was to make group activities transparent and visible for all team members. Team members can document finished and pending tasks for everyone in a central repository. In doing so, team communication remains accessible over time (e.g., for new employees) and changes in tasks are documented for different points in time. Moreover, for project leaders the platform is an effective medium for delegating and coordination.

Table 1 presents the four communication practices, which capture over 90\% of communication observed in the case team. It shows that EMB is well suited for coordinating teamwork on joint tasks. Thus team members post short status updates whenever they have finished working on a task or whenever problems arise. Team members document pending tasks on the platform and delegate tasks to other team members with a short and concise message, but in contrast to email is visible for all team members. Moreover, EMB is used to pose work-related questions, to reply to questions, to post guidelines or generally to solve problems. Another main team practice is to update other team members on events that happened outside the immediate team context (e.g., meeting with a customer). Finally, EMB is a medium for driving team discussions, e.g., by capturing ideas and posting links to interesting Internet sources.

The latter aspect points to potentials of EMB other than for immediate team coordination, such as for innovation management. Real innovation cannot be planned in advance. But EMB can create the necessary awareness for the activities of other people and facilitate the collection of ideas, which enables making contact with others and facilitating discussions. By doing so, seemingly "stupid" ideas can gain visibility and find unexpected promoters within the organization. 
Table 1 EMB Usage practices with examples (see Riemer and Richter 2010)

\begin{tabular}{|c|c|c|}
\hline Practice & Description & Example \\
\hline Task coordination & $\begin{array}{l}\text { Team members delegate pending } \\
\text { tasks to others, post lists of } \\
\text { pending tasks, report on finishing } \\
\text { tasks or enquire about task status. }\end{array}$ & $\begin{array}{l}\text { "Pricing for migration plugin \#pending" } \\
\text { "@mno is the current state ready for testing? } \\
\text { Where?" } \\
\text { "Just created a test installation on \#com } 233 \\
\text { and updated \#searchindex" }\end{array}$ \\
\hline Problem solving & $\begin{array}{l}\text { Team members ask work-related } \\
\text { questions, point out problems or } \\
\text { post guidelines and "how tos". }\end{array}$ & $\begin{array}{l}\text { "What is RAD studio able to do that Visual } \\
\text { Studio isn't?" } \\
\text { "Solved \#connectionproblem with \#sys. But } \\
\text { new problem came up. Configuration of. .." }\end{array}$ \\
\hline Event updates & $\begin{array}{l}\text { Team members report on events } \\
\text { outside the immediate team (e.g., } \\
\text { on contracts, phone calls, } \\
\text { meetings) and notify others of } \\
\text { upcoming events. }\end{array}$ & $\begin{array}{l}\text { "Received order from Klug Media Systems } \\
\text { \#KMS for \#Confluence development" } \\
\text { "Date for final migration 2009-08-17 @cde } \\
\text { @jkl" } \\
\text { "Just spoke to Mr. Klaus about the following } \\
\text { points..." }\end{array}$ \\
\hline $\begin{array}{l}\text { Ideas and information } \\
\text { input }\end{array}$ & $\begin{array}{l}\text { Team members post URLs to } \\
\text { interesting websites or offer ideas } \\
\text { for discussion. }\end{array}$ & $\begin{array}{l}\text { "for a first overview of Confluence 3.0: } \\
\text { http://confluence.atlassian.com/..." } \\
\text { "@tuv idea: The barrier could be placed } \\
\text { after the email address. Just like here..." }\end{array}$ \\
\hline
\end{tabular}

\section{Significance for the BISE Domain}

\subsection{Appropriation and Use}

Enterprise Microblogging resembles a socalled infrastructure technology. Such infrastructures are open, heterogeneous bundles of ICT-based potentials which allow users to establish usage practices (cf. Hanseth and Lytinnen 2004). Much like other communication and collaboration systems, EMB platforms are characterized by a form of openness which is best described by the German word "Nutzungsoffenheit". Such openness implies that these systems can be used in a wide variety of ways and that their potentials and effects in context cannot be deduced from examining their features and characteristics (Riemer and Taing 2009). Rather, potentials will only show up in appropriations by concrete users, e.g., as illustrated in the above case example.

In this context it is important to distinguish between practices of creation (i.e., the active posting of messages) and consumption (the use of the emerging information stream). While some studies, such as the above, have investigated active communication to a certain extent, so far no research exists on the role and usefulness of EMB as an information medium. It is, however, important to critically examine the claim made in practically ori- ented publications that EMB will solve or alleviate the information overload problem commonly associated with email usage. For example, it may well be that the undirected communication of information in a continuous EMB information stream will only lead to shifting the problem to this new medium. Quite possibly, this will to a considerable extent depend on the design of EMB platforms and (the appropriation of) features such as tagging and information filtering. It remains to be seen if the expectations held by platform providers and proponents of EMB regarding improvements in knowledge, innovation, and communication management will materialize. Currently, not enough case examples exist to make reliable predictions.

\subsection{Technical Developments and Integration}

Besides the above-discussed use, EMB also brings some technical challenges along. Among these are technical stability and availability, security, roles, and rights management as well as (based on the above-introduced notion of EMB as infrastructure) the integration with existing communication systems. In that respect, availability for communication in various communication channels becomes an important consideration (cf. Riemer and Taing 2009).
Moreover, it is important to view EMB as part of an ecosystem of existing services and systems and to take care of proper integration. Some initial works exist that have pointed to potentials for linking EMB with business processes and technical facilities and machinery. It has been suggested under the term ubiquitous microblogging that short messages, generated by non-human actors, be integrated with the EMB information stream and thus be made available to users in a unified medium (Böhringer and Gluchowski 2010). This again is related to the consumption part of microblogging, i.e., the question how information is consumed in this new medium. The assumption is that information supply can be simplified drastically if status updates by team members and those provided by processes and systems are made available in one integrated (filterable) information stream. Again, there is still a lack of empirical studies which investigate benefits and limits of such integration.

\subsection{Roll-Out and Management}

Enterprise Microblogging belongs to a range of other technologies that have been adapted for enterprise contexts, such as wikis, blogs, and social networking services. According to experiences made with these tools, it is to be expected that the challenges of using mi- 
croblogging in a corporate context are nor primarily technical in nature. In fact, some authors see a need for organizations that want to successfully apply the Twitter principle, to instigate a cultural and organizational change process which has been captured by the term Enterprise 2.0 (McAfee 2009). First studies suggest that EMB, more so than existing social software, will allow communication on equal terms, transparency, open communication across hierarchical levels and a new form of self presentation (for a discussion of social effects of Enterprise Microblogging, see Günther et al. 2009; Oulasvirta et al. 2009). However, it remains to be seen to what extent a deep organizational change process is actually needed, given that the openness of such platforms allows flexible adaptation to different situations.

Other studies in the Enterprise 2.0 domain (e.g., Richter and Koch 2009) suggest that the appropriation of such open platforms can be facilitated especially with the support of middle management and a concrete outlining of expected usage benefits. Future research should use this as a starting point for deriving ro- bust benefit measurements (e.g., for capturing ROI) and for critically questioning the suggested positive effects of an Enterprise 2.0 culture on corporate success. This, however, can only be achieved holistically by incorporating the qualitative aspects of social software application which are hard to quantify.

\section{References}

Barnes SJ, Böhringer M, Kurze C, Stietzel J (2010) Towards an understanding of social software: the case of Arinia. In: Proceedings of the 43rd Hawaii international conference on system sciences (HICSS-43)

Böhringer M, Gluchowski P (2010) Ubiquitous Microblogging als dezentrales Entwurfsparadigma für leichtgewichtige Informationssysteme: Nutzenpotenziale und Forschungsperspektiven. In: Tagungsband der Multikonferenz Wirtschaftsinformatik Göttingen

Böhringer M, Gerlach L, Lang A, Hauptmann S, Pham M (2010) Enterprise microblogging study 2010 (\#EMS10). http://ubimic.org/en/ enterprise-microblogging-study-ems 10/. Accessed 2010-04-06

Gartner (2010) Gartner reveals five social software predictions for 2010 and beyond. http://www.gartner.com/it/page.jsp?id= 1293114. Accessed 2010-04-06

Günther O, Krasnova H, Riehle D, Schönberg $\checkmark$ (2009) Modeling micro-blogging adop- tion in the enterprise. In: Proceedings of the 15th Americas conference on information systems (AMCIS 2009), San Francisco

Hanseth O, Lytinnen K (2004) Theorizing about the design of information infrastructures: design kernel theories and principles. Case Western Reserve University, USA. Sprouts Working Papers on Information Systems 12(4)

Java A, Song X, Finin T, Tseng B (2007) Why we twitter: understanding microblogging usage communities. In: Proceedings of the 9th WebKDD and 1st SNA-KDD 2007 workshop on web mining and social network analysis, pp 56-65

McAfee A (2009) Enterprise 2.0: new collaborative tools for your organization's toughest challenges. McGraw-Hill, Boston

Naaman M, Boase J, Lai C (2010) Is it really about me? Message content in social awareness streams. In: CSCW 2010, Savannah, USA

Oulasvirta A, Lehtonen E, Kurvinen E, Raento $M$ (2009) Making the ordinary visible in microblogs. Personal and Ubiquitous Computing 14(3):237-249

Richter A, Koch M (2009) Der Einsatz von Social Networking Services im Unternehmen. In: Proceedings 9. Internationale Tagung Wirtschaftsinformatik, Wien

Riemer K, Richter A (2010) Tweet inside: Microblogging in a corporate context. In: Proceedings 23 rd bled eConference eTrust: implications for the individual, enterprises and society, Bled

Riemer K, Taing S (2009) Unified communications. WIRTSCHAFTSINFORMATIK 51(4): 376-380 